\title{
ADHS, eine Herausforderung für Wissenschaft, Klinik und Praxis
}

Nur wenige Krankheitsbilder in der Psychiatrie werden so leidenschaftlich, vor allem von Nicht- Fachärzten, diskutiert wie die Aufmerksamkeitsdefizit-/Hyperaktivitätsstörung. Bereits in der psychiatrischen Literatur des 19. Jahrhunderts beschrieben, hat die therapeutische Beeinflussbarkeit seit den 30er Jahren des 20. Jahrhunderts die Grundlagen- aber auch die klinische Forschung angeregt. Allen, zum Teil mit jakobinischem Eifer, geführten Diskussionen und vor allem in antipsychiatrischen Kreisen zum Trotz wissen wir heute über Pathophysiologie, Epidemiologie, Klinik, Verlauf und therapeutische Beeinflussbarkeit der ADHS mehr als zu vielen anderen Störungsbildern in der Medizin. Das vorliegende Heft gibt einen guten Überblick über den aktuellen Wissensstand zur Ätiopathogenese (Beitrag von K.-H. Krause), zur sozialmedizinischen Relevanz (Beitrag von M. Rösler), zur psychotherapeutischen Beeinflussbarkeit (Beitrag von A. Philipsen) sowie zur Versorgung betroffener Patienten (Beitrag von M. Schlander). Die noch nicht ausreichende Akzeptanz dieser nosologi- schen Entität führt dazu, dass viele Patienten nicht von Kinder- und Jugendpsychiatern und Psychiatern versorgt werden und die Behandlung weder in Bezug auf Effektivität noch auf Effizienz optimal ist. Da die psychiatrische Komorbidität hoch ist, steht zu befürchten, dass gerade die komorbiden Störungen, die die Langzeitprognose ganz wesentlich beeinflussen, häufig übersehen werden.

Es hat sich viel im letzten Jahrzehnt getan. Auf den psychiatrischen und kinder- und jugendpsychiatrischen Kongressen wird der ADHS die gebührende Aufmerksamkeit eingeräumt. Der erste wissenschaftliche, internationale ADHSKongress fand im Juni in Würzburg statt. So bleibt zu hoffen, dass das Wissen und die Akzeptanz dieser die Lebensqualität erheblich beeinträchtigenden Störung in Klinik und Praxis schnell weiter fortschreiten wird und effektive, evidenzbasierte und ökonomisch vertretbare Behandlung dem Patienten zugutekommen. An dieser Stelle sei bemerkt, dass die wissenschaftlich fundierte und evidenzbasierte Behandlung zugleich die kostengünstigste ist.

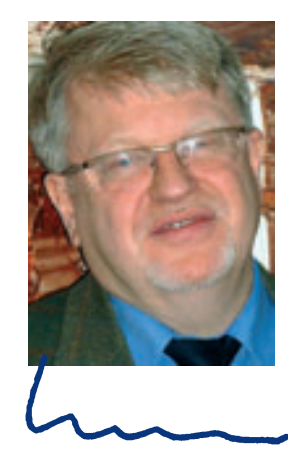

Prof. Dr. med. Götz-Erik Trott, Aschaffenburg 